\title{
EFEITO DA APLICAÇÃO DO POTÁSSIO, DO SÓDIO E DA DISPONIBILIDADE HÍDRICA NA DENSIDADE APARENTE A 12\% DE UMIDADE DO LENHO JUVENIL DE ÁRVORES DE Eucalyptus grandis
}

\author{
APPLICATION EFFECT OF POTASSIUM, SODIUM AND WATER AVAILABILITY IN THE \\ APPARENT DENSITY AT 12\% HUMIDITY OF JUVENILE WOOD OF Eucalyptus grandis TREES
}

\author{
Vinicius Resende Castro ${ }^{1}$ Paula Gabriella Surdi ${ }^{2}$ Carlos Roberto Sette Junior ${ }^{3}$ \\ Mario Tomazello Filho ${ }^{4}$ Gilles Chaix ${ }^{5}$ Jean Paul Laclau ${ }^{6}$
}

\begin{abstract}
RESUMO
Expressiva área das plantações florestais de eucalipto de rápido crescimento no Brasil e em outras regiões do mundo encontra-se sob o efeito das mudanças climáticas, do estresse hídrico e da baixa fertilidade do solo, afetando o crescimento e a qualidade do lenho das árvores. O presente trabalho teve como objetivo avaliar a densidade aparente a 12\% de umidade do lenho das árvores de Eucalyptus grandis com 12, 24 e 36 meses, com redução da precipitação pluviométrica e nutrição de $\mathrm{K}$ e Na. Os tratamentos foram definidos por dois regimes hídricos (100 e 66\% das chuvas, com redução artificial com lonas de polietileno) e três tipos de nutrição: $\mathrm{K}(\mathrm{K}-4,5 \mathrm{kmol} / \mathrm{ha}), \mathrm{N}$ (Na - 4,5 kmol/ha) e controle. A densidade aparente a $12 \%$ de U.R. das amostras da posição do DAP do tronco das árvores foi determinada pela densitometria de raios X e por imagens digitais do lenho. Os resultados indicaram diferença significativa da densidade aparente a $12 \%$ de U.R. das árvores de eucalipto nos tratamentos de nutrição e de disponibilidade hídrica. O perfil radial de densidade aparente mostrou maior diferença a partir do terceiro ano de idade das árvores. Pelas imagens digitais, analisou-se a variação radial da densidade aparente da madeira. Os resultados do trabalho fornecem subsídios para a adoção de práticas silviculturais nas áreas de plantações florestais com estresse hídrico e/ ou afetadas pelas mudanças climáticas, bem como a substituição parcial de K por Na.
\end{abstract}

Palavras-chave: nutrição; estresse hídrico; densitometria de raios X; qualidade do lenho.

\section{ABSTRACT}

Expressive area of forest plantations fast-growing of eucalyptus in Brazil and in other regions of the world is under the influence of climate change, water stress and low soil fertility, affecting the growth and the quality of the wood from the trees. This study aimed to evaluate the apparent density at $12 \%$ relative humidity of the wood of Eucalyptus grandis trees with 12, 24 and 36 months, with reduced rainfall and nutrition $\mathrm{K}$ and $\mathrm{Na}$. The treatments were defined by two water regimes $(100 \%$ and $66 \%$ of rainfall, with artificial reduction with polyethylene sheeting) and three types of nutrition: $\mathrm{K}(\mathrm{K}-4.5 \mathrm{kmol} / \mathrm{ha})$,

1 Engenheiro Florestal, Dr. em Recursos Florestais pela Escola Superior de Agricultura Luiz de Queiroz, Universidade de São Paulo, Rua Augusta Siqueira, 62, CEP 36570-000, Viçosa (MG), Brasil. vresende@gmail.com

2 Engenheira Florestal, Dr ${ }^{\mathrm{a}}$. em Recursos Florestais pela Escola Superior de Agricultura Luiz de Queiroz, Universidade de São Paulo, Rua Augusta Siqueira, 62, CEP 36570-000, Viçosa (MG), Brasil. paulasurdi@usp.br

3 Engenheiro Florestal, Dr., Professor do Departamento de Engenharia Florestal, Universidade Federal de Goiás, Campus Samambaia, Caixa Postal 131, CEP 74690-900, Goiânia (GO), Brasil. crsettejr@hotmail.com

4 Engenheiro Agrônomo, Dr., Professor Titular do Departamento de Ciências Florestais, Escola Superior de Agricultura Luiz de Queiroz, Universidade de São Paulo, Av. Pádua Dias, 11, CEP 13418-900, Piracicaba (SP), Brasil.mtomazel@usp.br

5 Engenheiro Florestal, Dr., Pesquisador do Centre de Recherche Agronomique Pour le Développement, Montpellier, Av. Pádua Dias, 11, CEP 13418-900, Piracicaba (SP), Brasil.gilles.chaix@cirad.fr

6 Engenheiro Florestal, Dr., Pesquisador do Centre de Recherche Agronomique Pour le Développement (CIRAD), Montpellier, Av. Pádua Dias, 11, CEP 13418-900, Piracicaba (SP), Brasil. laclau@cirad.fr

Recebido para publicação em 26/03/2015 e aceito em 27/01/2016 
$\mathrm{N}(\mathrm{Na}-4.5 \mathrm{kmol} / \mathrm{ha})$ and control. The apparent density of the samples of diameter at breast height was determined by densitometry profile from digital images of rays X. The results indicated a significant difference in nutrition treatments and water availability in relation to the density of the wood. The radial apparent density profiles showed higher differences in the wood from the third year of evaluation. Digital images of the wood obtained by X-rays allowed to analyze the variation of the apparent density of the wood. Work results may be used as subsidies for the adoption of silvicultural practices in forest plantations in areas with water stress and/or affected by climate change as well as the partial replacement of $\mathrm{K}$ by $\mathrm{Na}$.

Keywords: nutrition; induced water stress; x-ray densitometry; wood quality.

\section{INTRODUÇÃO}

No Brasil, as áreas de implantação dos programas de reflorestamento com eucalipto são, de modo geral, de baixa fertilidade, com deficiência de potássio e outros minerais e com deficit hídrico (GAVA, 1997; GONÇALVES et al., 2004). Nesse sentido, para a expansão das plantações florestais no país é determinante compreender a interação dos minerais, como o sódio e potássio e da disponibilidade hídrica, e o seu efeito na sazonalidade e no crescimento e da qualidade do lenho das árvores.

Por outro lado, a substituição total ou parcial do potássio por sódio em plantações florestais, segundo Marschner (1995), apresenta interesse prático e científico. As fontes de potássio, contendo sódio em sua composição, requerem menor energia no processo de purificação, permitindo com isso, uma redução no preço final dos fertilizantes. A literatura científica reporta que esses elementos minerais influenciam o balanço hídrico das árvores e aumentam a sua eficiência no uso da água, sob condições de estresse hídrico e, por consequência, na sua resistência aos períodos de seca (ALMEIDA et al., 2010).

Os trabalhos sobre o efeito dos fertilizantes minerais na densidade e características tecnológicas do lenho de árvores jovens de eucaliptos (1-3 anos) são escassos e os resultados disponíveis são controversos, indicando o aumento (VIGNERON et al., 1995; BOUVET et al., 1999), a diminuição (SHIMOYAMA; BARRICHELO, 1989; WASHUSEN et al., 2005) e resultados sem efeito na densidade da madeira com teor de umidade variando de 12 a 15\% (BAMBER et al., 1982; PEREIRA, 1990). Diversas pesquisas ressaltam a importância de estudos sobre o efeito da fertilização mineral nas propriedades anatômicas, físicas e mecânicas da madeira de árvores de eucalipto com elevada taxa de crescimento (SILVEIRA; MALAVOLTA, 2000; SETTE JUNIOR et al., 2013).

Em relação às consequências do estresse hídrico no crescimento das árvores, os resultados, da mesma forma, são incipientes e algumas pesquisas têm sido conduzidas em ambiente controlado (NGUGI et al., 2004; COOPMAN et al., 2008) visando avaliar a qualidade do lenho das árvores e, mais especificamente, a sua densidade aparente, expressa pela razão da sua massa e volume, na condição de $12 \%$ de umidade relativa da madeira (ABNT NBR 7190, 1997). Na determinação da densidade aparente do lenho, utilizamse, em geral, os métodos destrutivos não possibilitando a avaliação precisa de corpos de prova de pequenas dimensões, bem como, da densidade entre os anéis de crescimento. Mais recentemente, os métodos não destrutivos, como o radiográfico de atenuação de raios $\mathrm{X}$ apresentam vantagens na determinação da densidade aparente, como a otimização do tempo de análise, maior precisão e eficiência no processamento dos dados (ARIZAPANA-ALMOCINAD, 2013; SURDI et al., 2014). A densitometria de raios X constitui uma importante ferramenta de análise de caracterização da madeira, permitindo avaliar o efeito da deterioração do lenho das árvores de eucalipto provocada por fungos de podridão-branca, a detecção dos limites do cerne-alburno, do efeito do manejo florestal nas propriedades da madeira, a produção anual de biomassa, dentre outras propriedades da madeira e a relação com a sua estrutura anatômica (TOMAZELLO FILHO et al., 2008). Inúmeros autores têm aplicado essa metodologia, como Silva et al. (2004), Tomazello Filho et al. (2008), Benjamin (2006), Sette Junior et al. (2009), Henriques (2012), Arizapana-Almocinad (2013) e Knapic et al. (2014) avaliando a variação radial da densidade aparente do lenho de árvores de Eucalyptus grandis, de 2 a 20 anos, obtendo valor médio de 0,46 a $0,80 \mathrm{~g} / \mathrm{cm}^{3}$ na região da medula e da casca.

A técnica da radiografia digital aplica os fundamentos da densitometria de raios $\mathrm{X}$, propiciando a obtenção da imagem da estrutura interna da amostra de madeira e que se relaciona com a sua composição química, densidade e teor de umidade. Essa metodologia é empregada para detectar com precisão as variações de densidade intra e interanéis de crescimento e associadas com as condições climáticas, 
fixação do carbono, influência da fertilização, de práticas silviculturais e coma qualidade da madeira (MANNES et al., 2007; KEUNECKE et al., 2012; CHERUBINI et al., 2013, SURDI et al., 2014).

Pelo exposto, o presente trabalho teve como objetivo avaliar o efeito da aplicação do potássio e do sódio, da disponibilidade de água no solo na densidade aparente a $12 \%$ de umidade do lenho de árvores de Eucalyptus grandis aplicando o método radiográfico.

\section{MATERIAL E MÉTODOS}

\section{Caracterização da área, seleção e corte do lenho das árvores}

O experimento foi instalado na Estação Experimental de Ciências Florestais de Itatinga - SP, por meio do convênio do Departamento de Ciências Florestais da ESALQ/USP e do Centro de Cooperação Internacional em Pesquisa Agronômica para o Desenvolvimento (CIRAD) / França. O ensaio faz parte de um amplo grupo de pesquisas que compõe o Projeto Universal CNPq no 471808/2012-1"Mudanças climáticas globais: indução do estresse hídrico por exclusão de chuva e efeito no crescimento e formação e qualidade do lenho de árvores de Eucalyptus grandis com fertilização potássica e sódica".

O clima da região é caracterizado como mesotérmico úmido (Cwa), segundo Köppen, com precipitação média anual de $1635 \mathrm{~mm}$ e temperatura média de $16,2^{\circ} \mathrm{C}$ e $28,6^{\circ} \mathrm{C}$, nos meses mais frio e mais quente, respectivamente. O solo é do tipo latossolo vermelho amarelo distrófico, de textura média, com a litologia composta por arenito, formação Marília, do grupo Bauru. A análise do solo na fase de implantação do experimento foi realizada por Maquère (2008), caracterizando os atributos químicos até 6 metros de profundidade. Os resultados das análises indicaram o teor médio de $\mathrm{K}$ e $\mathrm{Na}$ trocável no solo de $0,02 \mathrm{cmolc}$ $\mathrm{kg}^{-1}$ até 5 centímetros de profundidade e, inferior a $0,01 \mathrm{cmolc} \mathrm{kg}^{-1}$ entre 0,05 e 6 metros de profundidade, demonstrando deficiências severas dos nutrientes em todo o perfil.

No período entre os anos de 1945 a 2010, a área de estudo foi ocupada por plantações de Eucalyptus saligna e de Eucalyptus grandis. A implantação do experimento foi realizada em maio de 2010, com o plantio de clone de Eucalyptus grandis da empresa Suzano Bahia Sul, no espaçamento 3 x $2 \mathrm{~m}$ em covas entre as touças da rotação anterior. A fertilização básica foi realizada no plantio para todos os tratamentos, sendo distribuída a lanço e sem incorporação no solo, a base de $2000 \mathrm{~kg}$ de calcário dolomítico/ha; posteriormente, aplicaram-se na cova os seguintes fertilizantes: $75 \mathrm{~kg}$ de $\mathrm{P}_{2} \mathrm{O}_{5}, 80 \mathrm{~kg}$ de N $\left(\mathrm{NH}_{4}\left(\mathrm{SO}_{4}\right)_{2}\right)$ e $20 \mathrm{~kg}$ de FTE (BR-12) por hectare.

Foi utilizado o delineamento experimental de blocos subdivididos, tipo split-plot, com 3 blocos e 6 tratamentos, totalizando 18 subparcelas. Os tratamentos compreenderam: (i) 2 regimes hídricos: 100\% e $66 \%$ das chuvas (exclusão artificial pela utilização de cobertura do solo de $1700 \mathrm{~m}^{2}$ de lonas de polietileno transparente) e (ii) 3 doses de fertilizantes: 0 (controle); $4,5 \mathrm{kmol} / \mathrm{ha}$ e $4,5 \mathrm{kmol} / \mathrm{ha}$ na forma de $\mathrm{KCl}$ e $\mathrm{NaCl}$, respectivamente, aplicados 3 meses, após o plantio. Para cada bloco, foram sorteados, inicialmente, os 2 tratamentos de regime hídrico (100 e $66 \%$ de chuvas) e, na sequência, os 3 tratamentos de nutrição (controle, $\mathrm{K}$ e Na), assim identificados: (a) $\mathrm{C} /+\mathrm{A}$, controle a $100 \%$ de chuva; (b) $\mathrm{Na} /+\mathrm{A}$, sódio a $100 \%$ de chuva; (c) $\mathrm{K} /+\mathrm{A}$, potássio a $100 \%$ de chuva; (d) $\mathrm{C} /-\mathrm{A}$, controle a $66 \%$ de chuva; (e) $\mathrm{Na} /-\mathrm{A}$, sódio a $66 \%$ de chuva; (f) K/-A, sódio a $66 \%$ de chuva.

O regime hídrico foi conduzido por redução/exclusão artificial de $34 \%$ da chuva, por meio de calhas confeccionadas com fios de arame que sustentavam as lonas de plástico. As calhas apresentavam uma largura de $40 \mathrm{~cm}$ e cobriam $34 \%$ da superfície total do solo (Figura 1) e foram apoiadas em estacas de madeira (altura de 1,5 a 0,30 m), em declive para a retirada da água das chuvas, a fim de reduzir o efeito da umidade do solo. A cada quinzena, eram retiradas as folhas e ramos retidos nas lonas plásticas, para fins de limpeza.

Para a determinação da densidade aparente a 12\% de umidade do lenho, foram selecionadas 4 árvores de eucalipto por tratamento (nutrição e disponibilidade hídrica), no $12^{\circ}, 24^{\circ}$ e $36^{\circ}$ mês de idade, totalizando 72 árvores. As árvores selecionadas nas 3 idades foram consideradas representativas da classe de área basal média do plantio. 

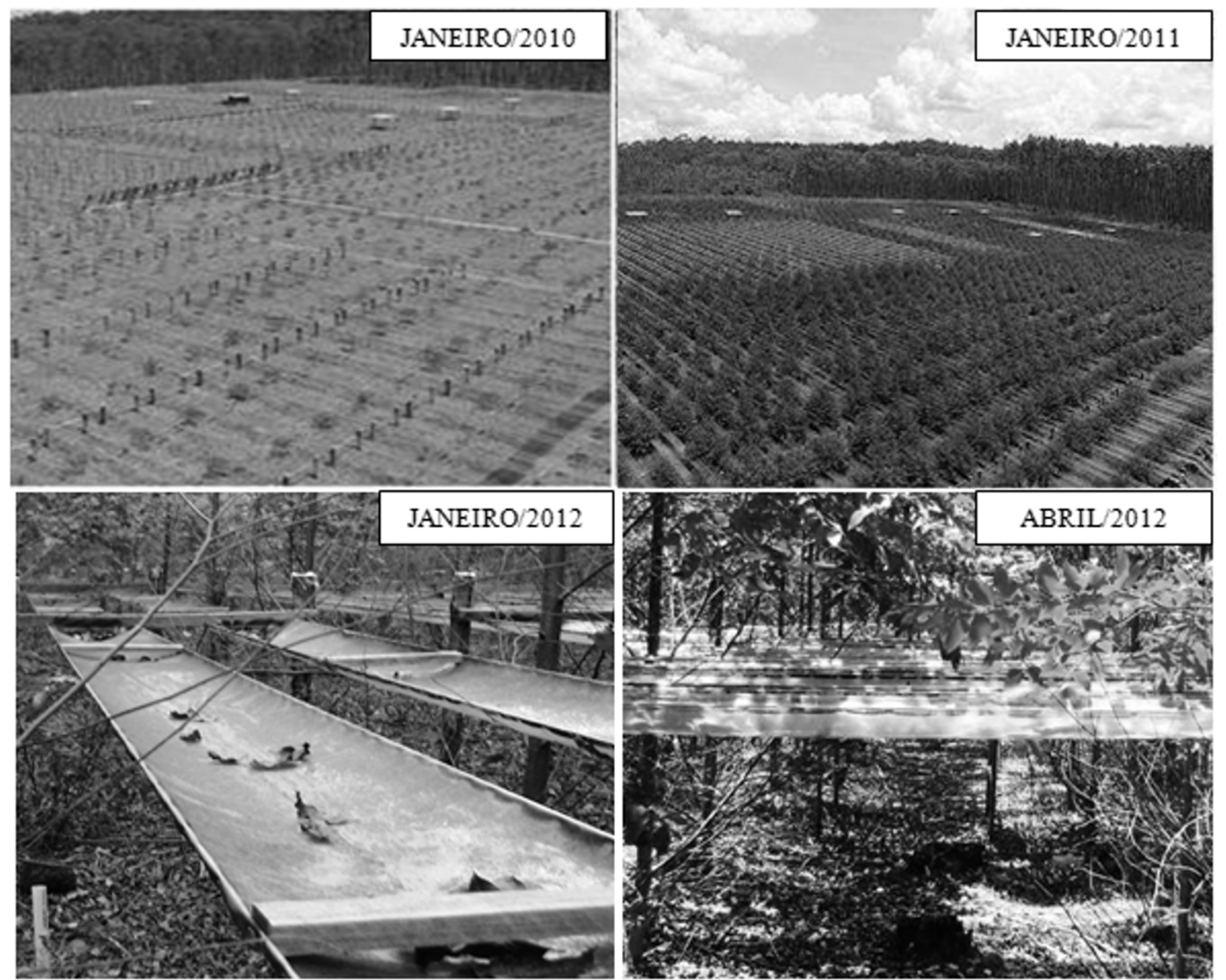

FIGURA 1: Aspecto do experimento e do delineamento das árvores de Eucalyptus grandis e detalhe das calhas com as lonas de polietileno, apoiadas em estacas de madeira.

FIGURE 1: Aspect of experiment and design of Eucalyptus grandis trees and detail of the rails with polyethylene sheeting, supported by wooden stakes.

\section{Obtenção dos perfis radiais de densidade aparente do lenho}

A partir das árvores selecionadas, foram seccionados discos do lenho a 1,3 m (DAP), nas 3 idades analisadas e posteriormente, foram cortadas amostras diametrais $(20 \times 10 \mathrm{~mm}$, largura x espessura) (Figuras 2A e B), coladas em suporte de madeira e seccionadas na direção radial, uma fina amostra com $2,0 \mathrm{~mm}$ de espessura em equipamento de dupla serra circular paralela (Figuras $2 \mathrm{C}$ e D). As seções radiais foram acondicionadas em câmara de climatização (vinte graus Celsius, por vinte e quatro horas, a sessenta por cento de umidade relativa do ar e a doze por cento de umidade relativa da madeira) (SURDI et al., 2014).

As amostras finas da seção radial do lenho e junto com a escala de calibração de acetato de celulose foram inseridas no compartimento blindado do equipamento de raios X digital Faxitron modelo LX-60 previamente calibrado para a leitura automática ( $30 \mathrm{Kv}, 19$ segundos). As imagens digitais com ultracontraste e resolução foram salvas em formato DICOM (FAXITRON, 2009) (Figuras 2E e F). Na montagem do perfil radial de densidade aparente a $12 \%$ de umidade da madeira, as imagens digitais em escala de cinza (Figura $2 \mathrm{G}$ ) e a escala de calibração foram analisadas no software ImageJ, para determinar os valores radiais de densidade aparente do lenho (a cada $50 \mu \mathrm{m}$ ), obtidos pelo software e transferidos para a planilha eletrônica, para a construção dos perfis diametrais de densidade aparente.

Para fins de comparação e a análise visual da superfície transversal do lenho, foram cortadas amostras gêmeas $(0,5 \mathrm{~cm}$ de espessura) do DAP do tronco das árvores de eucalipto nas 3 idades e 6 tratamentos, lixadas (lixa grão 180), escaneadas e acondicionadas $\left(20^{\circ} \mathrm{C}, 24 \mathrm{~h}, 60 \% \mathrm{UR}, 12 \%\right.$ de umidade relativa da madeira) para a obtenção das imagens digitais a partir da metodologia aplicada para as amostras finas do lenho (2 mm). 


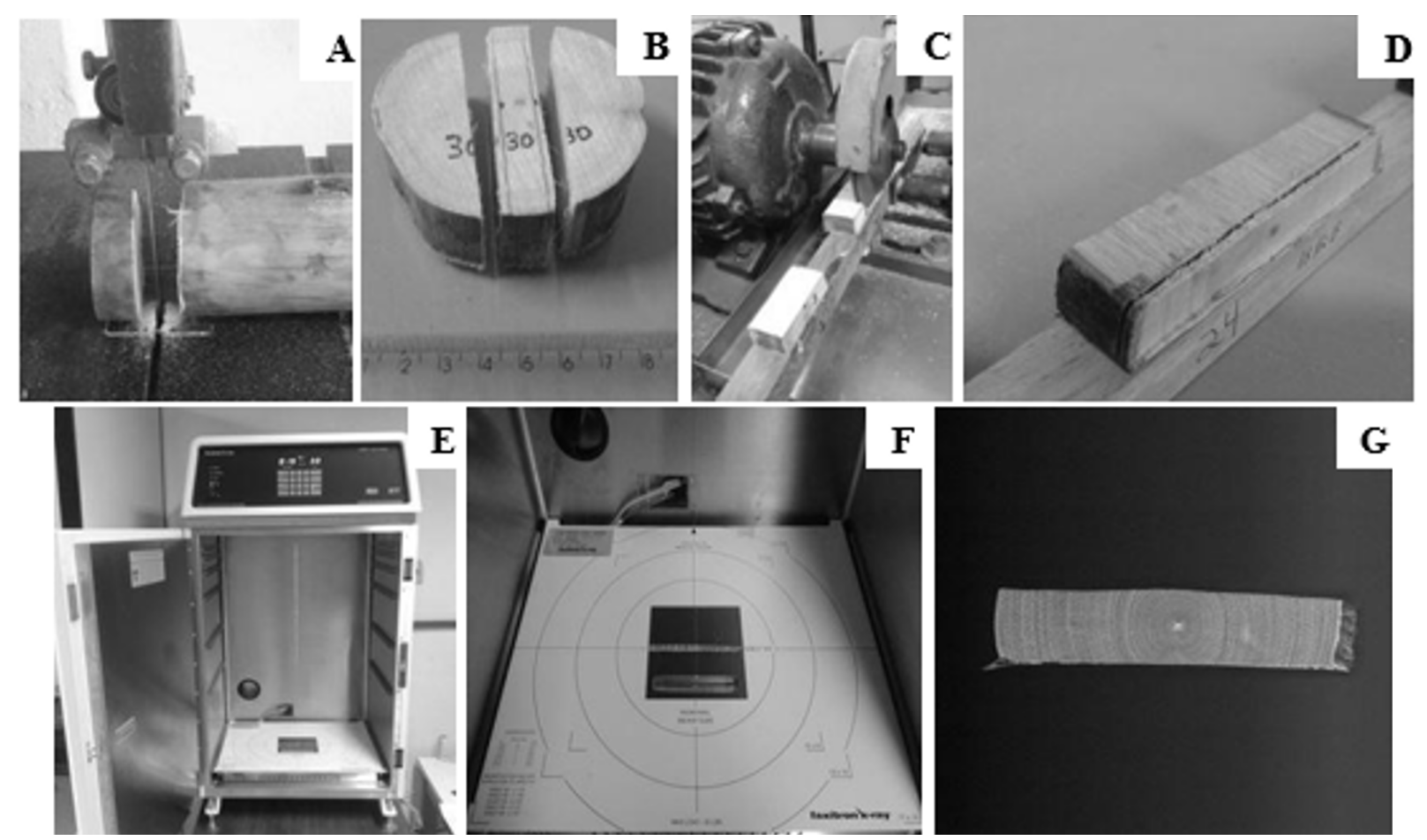

FIGURA 2: Obtenção das imagens digitais do lenho de Eucalyptus grandis em equipamento de raios $\mathrm{X}$ (A, B, C e D) corte e preparação da amostra transversal do lenho ( $2 \mathrm{~mm}$, espessura); (E, F) equipamento de raios X LX-60; (G) imagem digital do lenho.

FIGURE 2: Obtaining of the digital images of wood of Eucalyptus grandis in X-ray equipment (A, B, C and D) cutting and preparation of the wood sample (2 mm thickeness); (E, F) X-ray equipment LX-60; $(\mathrm{G})$ digital image of the wood.

Para verificar o efeito dos fatores nutrição (controle, Na e K) e disponibilidade hídrica (66 e 100\% de chuva) e sua interação (nutrição x disponibilidade hídrica) sobre a densidade aparente a $12 \%$ de umidade do lenho, foram aplicadas análises de variância (ANOVA), e, posteriormente, o teste de Tukey, ajustado a 95\% de probabilidade, com a utilização do programa SAS (SAS INSTITUTE, 1999).

Além da densidade aparente média, foi determinada a densidade aparente mínima e máxima, tendo como repetição os valores de mínimo e máximo de cada árvore analisada para cada tratamento nas três idades avaliadas.

\section{RESULTADOS E DISCUSSÃO}

O valor médio da densidade aparente do lenho a $12 \%$ de umidade no DAP do tronco das árvores, no primeiro e segundo ano de avaliação, não apresentou diferença entre os tratamentos (nutrição e disponibilidade hídrica), exceto para o tratamento $\mathrm{C} /+\mathrm{A}$, no $12^{\circ}$ mês. No primeiro ano de avaliação, a densidade aparente do lenho das árvores foi maior nos tratamentos nutrição/controle, com e sem redução de chuva, seguindo-se os tratamentos de aplicação de $\mathrm{K}$ e Na, independentemente da disponibilidade hídrica. No $24^{\circ}$ mês, verificou-se a mesma tendência entre tratamentos, embora, com valores mais homogêneos. No $36^{\circ}$ mês, observou-se a mesma tendência de valores de densidade entre os tratamentos, com maior densidade do lenho nos tratamentos nutrição/controle, com e sem redução de chuva, embora, estatisticamente similar ao tratamento $\mathrm{K} /$-A (Tabela 1 ).

A densidade aparente do lenho, mínima e máxima a 12\% de umidade, do tronco das árvores, nos 2 anos iniciais, nos 6 tratamentos (nutrição e disponibilidade hídrica), não apresentou diferença estatística, com variação de 0,19-0,39 e de 0,61-0,82 g/ $\mathrm{cm}^{3}$, respectivamente. Essa variação da densidade do lenho 
TABELA 1: Densidade aparente média, máxima e mínima das árvores de Eucalyptus grandis nas 3 idades, por tratamento de nutrição e de disponibilidade hídrica.

TABLE 1: Wood apparent density mean, maximum and minimum of Eucalyptus grandis trees in 3 ages analyzed by treatment of nutrition and water availability.

\begin{tabular}{|c|c|c|c|c|}
\hline \multirow[t]{2}{*}{ Tratamento } & \multirow{2}{*}{$\begin{array}{c}\text { Idade } \\
\text { (meses) }\end{array}$} & \multicolumn{3}{|c|}{ Densidade aparente da madeira $\left(\mathrm{g} / \mathrm{cm}^{3}\right)$ a $12 \%$ de U.R. } \\
\hline & & Média & Máxima & Mínima \\
\hline $\mathrm{C} /+\mathrm{A}$ & & $0,49 \mathrm{~b}( \pm 0,04)$ & 0,73 a $( \pm 0,06)$ & 0,33 a $( \pm 0,06)$ \\
\hline $\mathrm{Na} /+\mathrm{A}$ & & $0,42 \mathrm{a}( \pm 0,04)$ & 0,66 a $( \pm 0,11)$ & $0,25 \mathrm{a}( \pm 0,05)$ \\
\hline $\mathrm{K} /+\mathrm{A}$ & & $0,43 \mathrm{ab}( \pm 0,03)$ & 0,63 a $( \pm 0,04)$ & 0,27 a $( \pm 0,05)$ \\
\hline $\mathrm{C} /-\mathrm{A}$ & 12 & $0,41 \mathrm{a}( \pm 0,02)$ & 0,66 a $( \pm 0,05)$ & $0,24 \mathrm{a}( \pm 0,05)$ \\
\hline $\mathrm{Na} /-\mathrm{A}$ & & 0,37 a $( \pm 0,05)$ & 0,60 a $( \pm 0,05)$ & 0,19 a $( \pm 0,08)$ \\
\hline $\mathrm{K} /-\mathrm{A}$ & & $0,41 \mathrm{a}( \pm 0,03)$ & $0,62 \mathrm{a}( \pm 0,02)$ & $0,25 \mathrm{a}( \pm 0,07)$ \\
\hline $\mathrm{C} /+\mathrm{A}$ & & 0,48 a $( \pm 0,01)$ & 0,65 a $( \pm 0,04)$ & 0,35 a $( \pm 0,01)$ \\
\hline $\mathrm{Na} /+\mathrm{A}$ & & $0,45 \mathrm{a}( \pm 0,01)$ & $0,61 \mathrm{a}( \pm 0,04)$ & 0,30 a $( \pm 0,06)$ \\
\hline $\mathrm{K} /+\mathrm{A}$ & & 0,45 a $( \pm 0,02)$ & $0,62 \mathrm{a}( \pm 0,02)$ & 0,30 a $( \pm 0,06)$ \\
\hline $\mathrm{C} /-\mathrm{A}$ & 24 & 0,48 a $( \pm 0,01)$ & 0,65 a $( \pm 0,02)$ & 0,33 a $( \pm 0,02)$ \\
\hline $\mathrm{Na} /-\mathrm{A}$ & & 0,46 a $( \pm 0,02)$ & 0,68 a $( \pm 0,02)$ & $0,31 \mathrm{a}( \pm 0,03)$ \\
\hline $\mathrm{K} /-\mathrm{A}$ & & 0,46 a $( \pm 0,01)$ & $0,67 \mathrm{a}( \pm 0,08)$ & 0,31 a $( \pm 0,02)$ \\
\hline $\mathrm{C} /+\mathrm{A}$ & & $0,54 \mathrm{~b}( \pm 0,01)$ & $0,72 \mathrm{a}( \pm 0,04)$ & $0,39 \mathrm{~b}( \pm 0,01)$ \\
\hline $\mathrm{Na} /+\mathrm{A}$ & & 0,50 a $( \pm 0,01)$ & $0,71 \mathrm{a}( \pm 0,04)$ & $0,36 \mathrm{~b}( \pm 0,06)$ \\
\hline $\mathrm{K} /+\mathrm{A}$ & & 0,50 a $( \pm 0,02)$ & 0,82 a $( \pm 0,02)$ & 0,28 a $( \pm 0,06)$ \\
\hline $\mathrm{C} /-\mathrm{A}$ & 36 & $0,55 \mathrm{~b}( \pm 0,01)$ & $0,76 \mathrm{a}( \pm 0,02)$ & $0,37 \mathrm{~b}( \pm 0,02)$ \\
\hline $\mathrm{Na} /-\mathrm{A}$ & & $0,52 \mathrm{ab}( \pm 0,02)$ & 0,79 a $( \pm 0,02)$ & $0,34 \mathrm{ab}( \pm 0,03)$ \\
\hline $\mathrm{K} /-\mathrm{A}$ & & $0,54 \mathrm{~b}( \pm 0,01)$ & $0,80 \mathrm{a}( \pm 0,08)$ & $0,34 \mathrm{ab}( \pm 0,02)$ \\
\hline
\end{tabular}

Em que: Médias seguidas do desvio padrão expressas em $\left(\mathrm{g} / \mathrm{cm}^{3}\right)$. Médias seguidas da mesma letra, na coluna, por idade, não diferem entre si, pelo teste de Tukey $(\alpha=0,05)$.

relaciona-se com a estrutura anatômica do lenho caracterizada por regiões de fibras de parede espessa e menor frequência de vasos, de maior densidade, e de fibras mais delgadas e maior frequência de vasos de menor densidade, detectadas pela varredura da amostra do lenho pela fonte colimada de raios X, com leitura a cada $50 \mu \mathrm{m}$. Tal variação das características anatômicas e de formação do lenho foi avaliada por Freitas et al. (2015) no mesmo campo experimental, com as mesmas árvores analisadas no presente estudo.

$\mathrm{O}$ valor da densidade aparente média do lenho das árvores de eucalipto no $12^{\circ}, 24^{\circ}$ e $36^{\circ}$ mês foi inferior à determinada para a espécie, por inúmeros autores, como Silva et al. (2004), Tomazello Filho et al. (2008), Benjamin, (2006), Sette Junior et al. (2009), Henriques (2012), Arizapana-Almocinad (2013) e Knapic et al. (2014), aplicando a mesma metodologia de raios X e com mesmo teor de umidade (12\% de umidade da madeira), sendo relacionada com a diferença da idade das árvores e associada à formação de madeira juvenil-adulta.

Os resultados das pesquisas sobre o efeito dos fertilizantes minerais nas propriedades da madeira de árvores adultas (até 30 anos) de eucalipto indicam o aumento (VIGNERON et al., 1995; BOUVET et al., 1999) e a redução da densidade do lenho (SHIMOYAMA; BARRICHELLO, 1989; WASHUSEN et al., 2005). Para as árvores jovens de eucaliptos (1-4 anos) destacam-se os resultados de Bamber et al. (1982), Pereira e Araújo (1990) e Sette Júnior et al. (2009; 2010), nos quais os autores verificaram que a fertilização mineral não diferenciou estatisticamente a densidade aparente do lenho.

Para as árvores de eucalipto no $12^{\circ}$ e $36^{\circ}$ mês após o plantio, a análise da variância mostrou diferença 
para o tratamento de nutrição e disponibilidade hídrica, embora sem interação entre os fatores. Nas árvores de eucalipto com 24 meses verificou-se diferença estatística apenas para o tratamento de nutrição. Dessa forma, o tratamento de nutrição mineral influenciou a densidade aparente do lenho das árvores de eucalipto no $12^{\circ}, 24^{\circ}$ e $36^{\circ}$ mês e a disponibilidade hídrica no $12^{\circ}$ e $36^{\circ}$ mês.

Nos tratamentos de nutrição foram verificados, através do teste de médias, que a aplicação de K e $\mathrm{Na}$ induziu a formação de lenho nas árvores de eucalipto de maior homogeneidade em relação ao parâmetro densidade aparente. No tratamento-controle observou-se o valor mais alto de densidade aparente do lenho das árvores de eucalipto, seguido do tratamento com $\mathrm{K}$ e Na (Tabela 2). No presente trabalho foi observado, dessa forma, a redução da densidade aparente do lenho em resposta à aplicação de fertilizantes, sendo que a literatura indica, também, que a nutrição mineral pode induzir o aumento da densidade do lenho.

TABELA 2: Densidade aparente média do lenho, nas três idades das árvores de eucaliptos, por tratamento de nutrição e de disponibilidade hídrica.

TABLE 2: Mean apparent wood density, at the three ages of eucalypts trees by treatment of nutrition and water availability.

\begin{tabular}{|c|c|c|c|}
\hline \multirow{2}{*}{ Tratamento } & \multicolumn{3}{|c|}{ Densidade aparente média do lenho $\left(\mathrm{g} / \mathrm{cm}^{3}\right)$ a $12 \%$ de umidade } \\
\hline & 12 meses & 24 meses & 36 meses \\
\hline Controle & $0,46 \mathrm{~b} \quad( \pm 0,06)$ & $0,48 \mathrm{~b}( \pm 0,01)$ & $0,54 \mathrm{~b}( \pm 0,01)$ \\
\hline $\mathrm{Na}$ & $0,39 \mathrm{a} \quad( \pm 0,05)$ & 0,46 a $( \pm 0,01)$ & $0,51 \mathrm{a}( \pm 0,02)$ \\
\hline $\mathrm{K}$ & $0,42 \mathrm{ab}( \pm 0,03)$ & 0,46 a $( \pm 0,02)$ & $0,52 \mathrm{a}( \pm 0,03)$ \\
\hline $100 \%$ & $0,45 \mathrm{~b} \quad( \pm 0,05)$ & $0,46 \mathrm{a}( \pm 0,01)$ & $0,52 \mathrm{a}( \pm 0,02)$ \\
\hline $66 \%$ & $0,40 \mathrm{a} \quad( \pm 0,04)$ & 0,46 a $( \pm 0,02)$ & $0,54 \mathrm{~b}( \pm 0,02)$ \\
\hline
\end{tabular}

Em que: Médias seguidas do desvio padrão expressas em $\left(\mathrm{g} / \mathrm{cm}^{3}\right)$. Médias seguidas da mesma letra, na coluna, por idade, não diferem entre si, pelo teste de Tukey $(\alpha=0,05)$.

Para as árvores de eucalipto com 12 e 24 meses, no tratamento de disponibilidade hídrica - analisado isoladamente - foi observado que o valor da densidade aparente média do lenho foi similar a todas as árvores do tratamento de nutrição e de disponibilidade hídrica. Nas árvores de eucalipto com 36 meses, o maior valor médio de densidade do lenho foi determinado no tratamento com redução de chuva. Oliveira et al. (2010) observaram, também, o aumento da densidade do lenho avaliando tratamentos de regimes de precipitação, com maior valor nos sítios caracterizados pela menor precipitação.

Na seção transversal do lenho identificou-se o primeiro anel de crescimento distinto, formado no $24^{\circ}$ mês e representativo da idade cambial das árvores de eucalipto (Figura 3). Na região do lenho do tronco das árvores de eucalipto com redução de chuva, correspondente a $85-100 \%$ do seu raio, foi detectada a maior variação da densidade aparente do lenho, referente ao lenho formado no $24^{\circ}$ e $36^{\circ}$ mês. As árvores de eucalipto submetidas aos tratamentos de estresse hídrico, independentemente do tratamento de nutrição, apresentaram a maior variação da densidade aparente do lenho.

O modelo de variação radial da densidade aparente do lenho, nas três idades, por tratamento de nutrição e de disponibilidade hídrica, indicou o menor valor $\left(0,35-50 \mathrm{~g} / \mathrm{cm}^{3}\right)$ na região próxima da medula, aumento e estabilização $\left(0,50-0,55 \mathrm{~g} / \mathrm{cm}^{3}\right)$ na região intermediária e aumento $\left(0,55-0,70 \mathrm{~g} / \mathrm{cm}^{3}\right)$ no lenho externo, próximo à casca. Assim, o perfil radial de densidade aparente do lenho indica (i) aumento da densidade do lenho no sentido medula-casca, (ii) formação da madeira com características de juvenilidade e (iii) zonas fibrosas com variação intra e interanual da densidade, como resultado da sazonalidade da atividade cambial e resposta às variações climáticas, de acordo com Tomazello Filho et al. (2008). Para as árvores de eucalipto, nas 3 idades, o perfil densitométrico evidenciou diferença com o obtido para as árvores adultas dessa espécie, de acordo com diversos autores, como Silva et al. (2004), Tomazello Filho et al. (2008), Benjamin (2006), Sette Junior et al. (2009); Couto et al. (2012), Henriques (2012), ArizapanaAlmocinad (2013) e Knapic et al. (2014). No entanto, é importante a avaliação contínua do lenho das árvores 
de eucalipto visando detectar as alterações na sua estrutura anatômica, densidade e demais propriedades, nos tratamentos de fertilização de $\mathrm{K}$ e $\mathrm{Na}$ e de restrição hídrica, bem como a caracterização da madeira para diferentes usos.
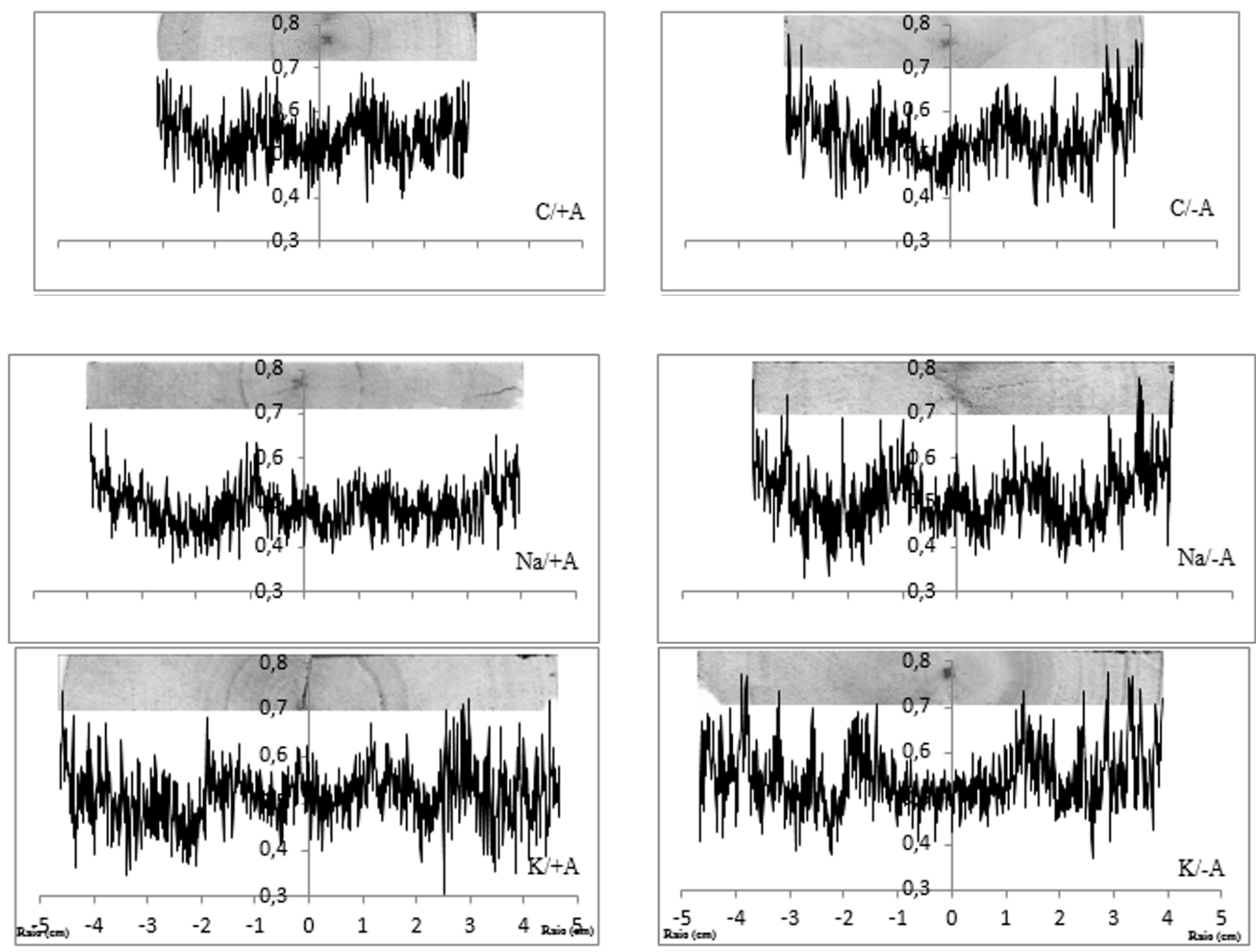

FIGURA 3: Perfil radial da densidade aparente do lenho no DAP do tronco das árvores de Eucalyptus grandis, no $36^{\circ}$ mês, nos tratamentos de nutrição e de disponibilidade hídrica.

FIGURE 3: Wood radial apparent density at the DBH of Eucalyptus grandis trees, 36th month of the treatment of nutrition and water availability.

A análise macroscópica da seção transversal do lenho no DAP do tronco das árvores de Eucalyptus grandis, nas três idades, não evidenciou as regiões de cerne e alburno, embora o xilema funcional esteja restrito à região próxima da camada cambial (Figura 4). No entanto, as imagens digitais da seção transversal das amostras do lenho das árvores de Eucalyptus grandis, com 24 e 36 meses, obtidas pelo equipamento de raios $\mathrm{X}$, indicaram a formação de zonas fibrosas distintas (faixas de crescimento) principalmente nos tratamentos de redução de chuva e de Na/-Ae K/-A. As faixas de crescimento mais claras, de maior atenuação dos raios $\mathrm{X}$ e de maior densidade aparente, têm a sua estrutura anatômica caracterizada pelas camadas de fibras de parede celular espessa e de vasos de menor frequência e diâmetro (Figura 4). A utilização das imagens digitais do lenho das árvores por irradiação de raios X possibilita, da mesma forma, a demarcação e a mensuração da largura dos anéis de crescimento constituindo-se em importante ferramenta para a dendrocronologia, conforme discutido por Cherubini et al. (2013). A formação dos anéis de crescimento anuais no lenho das árvores de eucalipto, segundo Argent et al. (2004), é resultado da variação da atividade cambial, afetada pela disponibilidade hídrica e temperatura ao longo das estações do ano. 


\begin{tabular}{lll}
\hline & 12 meses \\
\hline & \\
\hline & \\
\hline & \\
\hline
\end{tabular}
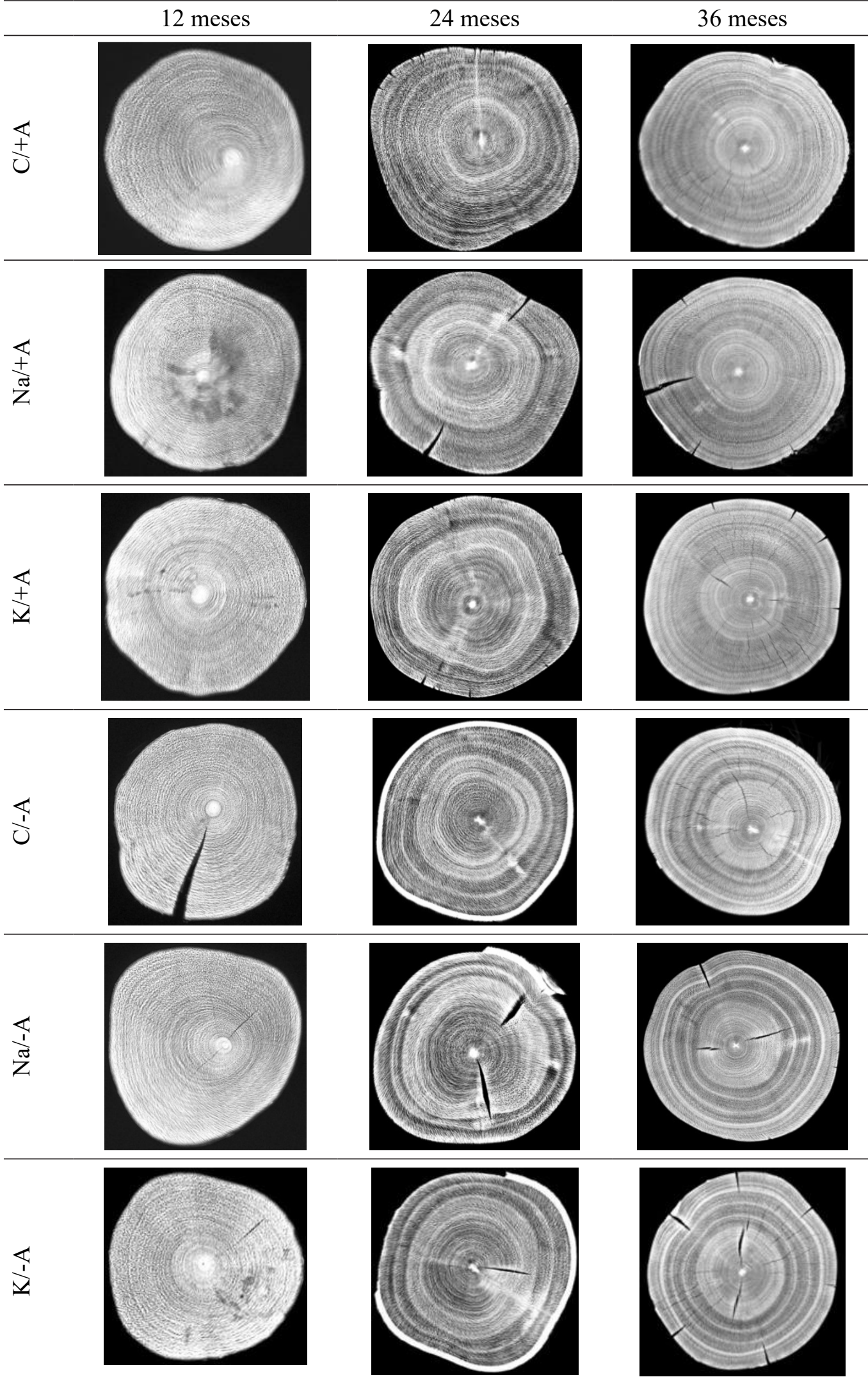

FIGURA 4: Imagem digital da seção transversal do lenho das árvores de Eucalyptus grandis, nas três idades, obtidas pela exposição aos raios X.

FIGURE 4: Digital image of the wood cross section of the Eucalyptus grandis trees at the three ages, by the exposition of X-rays source. 


\section{CONCLUSÕES}

Os resultados do presente trabalho permitiram concluir que: (i) a nutrição mineral influenciou a densidade aparente a 12\% de umidade do lenho das árvores de Eucalyptus grandis nas idades de 12, 24 e 36 meses e a disponibilidade hídrica no $12^{\circ}$ e $36^{\circ}$ mês; (ii) a aplicação do K e do Na resultou na formação do lenho das árvores de maior homogeneidade em termos da sua densidade aparente a $12 \%$ de umidade; (iii) no tratamento de disponibilidade hídrica, analisado isoladamente, a densidade aparente média do lenho das árvores no $12^{\circ}$ e $24^{\circ}$ mês, foi similar à observada no tratamento de nutrição e de disponibilidade hídrica; (iv) as árvores no $36^{\circ}$ mês, com redução da disponibilidade hídrica apresentaram maior valor de densidade aparente a $12 \%$ de umidade do lenho, indicando que o estresse hídrico aumenta a densidade da madeira, e (v) a metodologia pode ser aplicada na avaliação das práticas silviculturais em plantações florestais em áreas com estresse hídrico e na substituição parcial do K por Na.

\section{REFERÊNCIAS}

ALMEIDA, J. C. R. et al. A positive growth response to $\mathrm{NaCl}$ applications in Eucalyptus plantations established on K-eficient soils. Forest Ecology and Management, Amsterdam, v. 259, p. 1786-1795, 2010. ARIZAPANA-ALMONACID, M. A. Avaliação do crescimento e da qualidade do lenho de árvores de Eucalyptus urophylla, de 8 anos, de teste de progênies, visando a produção de carvão vegetal. 2013. 159 f. Dissertação (Mestrado em Recursos Florestais) - Escola Superior de Agricultura "Luiz de Queiroz", Universidade de São Paulo, Piracicaba, 2013.

ARGENT, R. A. et al. The dendroecological potential of Eucalyptus camaldulensis Denhardt (river red gum) from the Barmah Forest, Victoria, Australia. Australian Geographical Studies, Sydney, v. 42, n. 1, p 89-102, 2004.

ASSOCIAÇÃO BRASILEIRA DE NORMAS TÉCNICAS. NBR 7190: projeto de estruturas de madeira. Rio de Janeiro, 1997.

BAMBER, R. K. et al. Effect of fast growth on the wood properties of Eucalyptus grandis. Australian Forestry Research, Canberra, v. 12, n. 2, p. 163-167, dec. 1982.

BENJAMIN, C. A. Estudo da estrutura anatômica e das propriedades físicas e mecânicas da madeira de Corymbia (Eucalyptus) e Eucalyptus grandis. 2006. 158 f. Tese (Doutorado em Energia na Agricultura) - Faculdade de Ciências Agronômicas, Universidade Estadual Paulista "Júlio Mesquita Filho", Botucatu, 2006.

BOUVET, J. M. et al. Genetic and environmental effects on growth and wood basic density with two Eucalyptus hybrids. In: CONGRESS ON CONNECTING BETWEEN SILVICULTURE AND WOOD QUALITY, 1999, Nancy. Proceedings... Nancy: IUFRO, 1999. p. 15-19.

CHERUBINI, P. et al. Olive tree-ring problematic dating: a comparative analysis on Santorini (Greece). Plos One, Berkeley, v. 8, n. 1, p. 1-5, 2013.

COOPMAN, R. E. et al. Changes in morpho-physiological attributes of Eucalyptus globulus plants in response to different drought hardening treatments. Electronic Journal of Biotechnology, Valparaiso, v. 11, n. 2 , p. 30-39, 2008.

COUTO, A. M. et al. Validation of a X-Ray densitometry method for the determination of Eucalyptus grandis and Eucalyptus urophylla radial density profile. In: IUFRO CONFERENCE DIVISION 5 FOREST PRODUCTS, 2012, Estoril. Final program, proceedings and abstracts book... Estoril: IUFRO, 2012.

FAXITRON. User manual Faxitron D X radiography system. Lincolnshire: [s. n.], 2009. 95 p.

FREITAS, P. C. et al. Efeito da disponibilidade hídrica e da aplicação de potássio e sódio nas características anatômicas do lenho juvenil de Eucalyptus grandis. Revista Árvore, Viçosa, MG, v. 39, n. 2, p. 405-416, 2015.

GAVA, J. L. Efeito de adubação potássica em plantios de Eucalyptus grandis conduzidos em segunda rotação em solos com diferentes teores de potássio trocável. Série Técnica IPEF, Piracicaba, v. 11, n. 30, p. 89-94, abr. 1997.

GONÇALVES, J. L. M. et al. An evaluation of minimum and intensive soil preparation regarding fertility and tree nutrition. In: GONÇALVES, J. L. M.; BENEDETTI, V. (Ed.). Forest nutrition and fertilization. 
Piracicaba: IPEF, 2004. p. 13-64.

HENRIQUES, E. P. Variabilidade genética de caracteres de produção e qualidade da madeira, para carvão vegetal, em progênies de Eucalyptus urophylla S. T. Blake. 2012. 84 f. Dissertação (Mestrado em Ciência Florestal) - Universidade Estadual Paulista "Júlio de Mesquita Filho", Botucatu, 2012.

KEUNECKE, D. et al. Combination of X-ray and digital image correlation for the analysis of moistureinduced strain in wood: opportunities and challenges. European Journal of Wood and Wood Products, Berlin, v. 70, p. 407-413, 2012.

KNAPIC, S. et al. Early assessment of density features for 19 Eucalyptus species using X-ray microdensitometry in a perspective of potential biomass production. Wood Science and Technology, New York, v. 48, p. 37-49, 2014.

MAQUĖRE, V. Dynamics of mineral elements under a fast-growing/Eucalyptus/plantations in Brazil: implications for soil sustainability. 2008. 375 f. Thesis (Ph. D. in Forest Science) - Agro Paris Tech, Paris, 2008.

MANNES, D. et al. Neutron imaging versus standard X-ray densitometry as method to measure tree-ring wood density. Trees, Berlin, v. 21, p. 605-612, 2007.

MARSCHNER, H. Mineral nutrition of higher plants. San Diego: Academic Press, 1995. 889 p.

NGUGI, M. R. et al. Physiological responses to water stress in Eucalyptus cloeziana and E. argophloia seedlings. Trees, Darmstadt, v. 18, p. 381-389, 2004.

OLIVEIRA, J. T. S. et al. Influência da precipitação na densidade básica da madeira de um clone de Eucalyptus grandis. In: ENCONTRO BRASILEIRO EM MADEIRAS E EM ESTRUTURAS DE MADEIRA, 2010, Lavras. Anais... Lavras, 2010.

PEREIRA, A. R. Biomassa e ciclagem de nutrientes minerais em povoamentos jovens de Eucalyptus grandis e E. urophylla em região de cerrado. 1990.167 f. Tese (Mestrado em Ciências Florestais) Universidade Federal de Viçosa, Viçosa, 1990.

SETTE JUNIOR, C. R. et al. Efeito da aplicação de potássio e sódio nas características do lenho de árvores de Eucalyptus grandis W. Hill, aos 24 meses de idade. Revista Floresta, Curitiba, v. 39, n. 3, p. 535-546, jul./set. 2009.

SETTE JUNIOR, C. R. et al. Crescimento em diâmetro do tronco das árvores de Eucalyptus grandis W. Hill ex Maiden e relação com as variáveis climáticas e fertilização mineral. Revista Árvore, Viçosa, MG, v. 43, n. 6, p. 979-990, 2010.

SETTE JUNIOR, C. R. et al. Source-driven remobilizations of nutrients within stem wood in Eucalyptus grandis plantations. Trees, Heidelberg, v. 27, p. 827-839, 2013.

SHIMOYAMA, V. R. S.; BARRICHELO, L. E. G. Importância da adubação na qualidade da madeira e celulose. In: SIMPÓSIO SOBRE ADUBAÇÃO E QUALIDADE DOS PRODUTOS AGRÍCOLAS, 1989, Ilha Solteira. Anais... Ilha Solteira: 1989. p. 1-15.

SILVA, J. C. et al. Influência da idade e da posição radial na massa específica da madeira de Eucalyptus grandis Hill ex. Maiden. Revista Floresta, Curitiba, v. 34, n. 1, p. 13-22, jan./abr. 2004.

SILVEIRA, R. L. V. A.; MALAVOLTA, E. Nutrição e adubação potássica em Eucalyptus. Piracicaba: POTAFOS, 2000. 12 p. (Informações Agronômicas, 91 - Encarte Técnico, 12).

SURDI, P. G. et al. Perfil de densidade do lenho utilizando métodos radiográficos. Scientia Forestalis, Piracicaba, v. 42, p. 229-236, 2014.

TOMAZELLO FILHO, M. et al. Application of technique in nondestructive evaluation of eucalyptus wood. Maderas: Ciencia y Tecnologia, Concepción, v. 10, n. 2, p. 139-150, 2008.

VIGNERON, J. et al. Relationship between basic density and growth in a fertilization trial with clones of Eucalyptus hybrids in Congo. In: IUFRO CONFERENCE EUCALYPT PLANTATIONS: IMPROVING FIBRE YIELD AND QUALITY, 1995, Hobart. Proceedings... Hobart: IUFRO, 1995. p. 68-71.

WASHUSEN, R. et al. A. Effect of thinning and fertilizer on the cellulose crystallite width of Eucalyptus globulus. Wood Science and Technology, Berlin, v. 39, n. 7, p. 569-578, Oct. 2005. 